\title{
Assessment of the Effect of the Go Red for Women Campaign on Search Engine Queries for Cardiovascular Disease in Women
}

\author{
Giselle A. Suero-Abreu ${ }^{\mathrm{a}, \mathrm{d}}$, Aldo Barajas-Ochoa ${ }^{\mathrm{a}}$, Arturo Perez-Peralta ${ }^{\mathrm{a}}$, \\ Edward Rojas ${ }^{\mathrm{b}}$, Robert Berkowitz ${ }^{\mathrm{c}}$
}

\begin{abstract}
Background: The Go Red for Women (GoRedW) campaign aims to increase awareness of cardiovascular disease (CVD) and stroke in women. However, assessing the effects of social campaigns on information-seeking behaviors may be challenging. The purpose of this study was to ascertain the effect of GoRedW using a large sample of unbiased real-world data from Google Trends (GTr) and evaluate the temporal correlation of online search queries for CVD and stroke in women with GoRedW.
\end{abstract}

Methods: We conducted a retrospective study using GTr, a public tool from the Google search engine to obtain relative search volumes (RSVs) related to CVD and stroke in women in the period January 2004 to April 2019 in the USA. In addition, trends of GoRedW were compared with those of the well-established Breast Cancer Awareness Month (BCAM) campaign.

Results: RSVs increased for queries of GoRedW and all searched terms for CVD but not for stroke in women during February compared to other months of the year without active campaign. The strong pattern with peaks of temporal increase was consistent over the 15-year study period. RSV of "Go Red for Women" in February increased on average $494 \%$ (range: $211 \%$ to $789 \%$ ). The highest temporal increase on search queries for CVD was for the term "heart disease (in) women" with an average of $114 \%$ ( $24 \%$ to $182 \%)$. We found a positive correlation between RSVs of GoRedW and the term "heart disease (in) women" ( $\mathrm{r}$ $=0.54, \mathrm{P}<0.001)$. RSVs for "Breast Cancer Awareness Month" had a higher increase during the active campaign month compared to GoRe-

Manuscript submitted May 22, 2020, accepted June 15, 2020

Published online August 1, 2020

aDepartment of Medicine, Rutgers New Jersey Medical School, 150 Bergen Street, Newark, NJ 07103, USA

${ }^{b}$ Division of Cardiovascular Medicine, University of Virginia, 200 Jeanette Lancaster Way, Charlottesville, VA 22903, USA

'Heart Failure and Pulmonary Hypertension Program, Hackensack University Medical Center, 30 Prospect Ave, Hackensack, NJ 07601, USA

${ }^{\mathrm{d} C o r r e s p o n d i n g ~ A u t h o r: ~ G i s e l l e ~ A . ~ S u e r o-A b r e u, ~ D e p a r t m e n t ~ o f ~ M e d i c i n e, ~}$ Rutgers New Jersey Medical School, 150 Bergen Street, Newark, NJ 07103, USA. Email: giselle.suero@gmail.com

doi: https://doi.org/10.14740/cr1107
$\mathrm{dW}$ and showed a stronger positive correlation $(\mathrm{r}=0.78, \mathrm{P}<0.001)$.

Conclusions: Search engines are a valuable resource to provide insights on information demand and to assess the effectiveness of social campaigns and interventions. Our study showed an increase in the RSVs for queries of GoRedW and all CVD terms which correlated with the active campaign months over a 15 -year period.

Keywords: Health informatics; Cardiovascular disease; Epidemiology; Go Red for Women; Breast Cancer Awareness Month; Infodemiology; Cardio-oncology; Onco-cardiology

\section{Introduction}

In the USA, cardiovascular disease (CVD) remains the leading cause of mortality in women, exceeding that of cancer [1]. The Go Red for Women (GoRedW) campaign is an iconic initiative launched in 2004 with the aim to develop a strategic and integrated approach to the care of women and to increase awareness of CVD in women. Despite remarkable success of GoRedW over the past 15 years, awareness remains suboptimal especially among young women and racial and ethnic minority communities [2-4]. Patients often use Internet search engines to gather information on diseases, and such query data may provide an important tool for research, public health policies and to assess the impact of health campaigns such as GoRedW [5-8]. This is the framework of the emerging field of infodemiology that studies the determinants and distribution of health information on electronic media [6-8]. To date, there is no evaluation of the effect of GoRedW on online information seeking, such as the volume of Internet search queries related to CVD in women. The purpose of this research was to use Google Trends (GTr) to obtain demand-based infodemiology indicators in order to assess the effect of the GoRedW on search engine queries for CVD in women.

\section{Materials and Methods}

We assessed whether there was an association of GoRedW 
with searches for terms related to CVD and stroke in women. In addition, trends of GoRedW were compared with those of the well-established Breast Cancer Awareness Month (BCAM) campaign. Google is the most used search engine, with $91.98 \%$ of the search engine market share worldwide and $88.3 \%$ in the USA [9]. GTr is a web-based tool that provides access to an unbiased sample of actual search queries made by Google, which analyzes the popularity of searches queries, and visually displays them. GTr normalizes the search volumes of one or more terms to a scale of $0(<1 \%$ of peak volume) to 100 (peak popularity), and presents results as a relative search volume (RSV) $[8,10]$.

In this study, the primary objective was to assess whether there was a temporal association of the GoRedW campaign with the search volume of terms related to CVD in woman, as a surrogate marker of the campaign's effectiveness in promoting information seeking behavior and awareness on the topic. GTr data for the USA from January 2004 to April 2019 were analyzed. For GoRedW, the search terms "Go Red for Women", "heart attack in women," "heart attack women," "stroke in women," "stroke women," "heart disease in women," and "heart disease women" were queried in GTr and their RSVs were obtained. The RSVs for similar terms (e.g., "stroke in women" and "stroke women," hereafter written as "stroke (in) women") were aggregated. For BCAM, the terms "Breast Cancer Awareness Month" and "breast cancer" were used.

For each campaign, the search term's RSV during its active month (February for GoRedW; October for BCAM) was compared with the average of the other 11 months. The Pearson correlation coefficient between the RSV of each campaign and its related search terms was calculated. To estimate the effect of GoRedW relative to BCAM, each campaign's title and their respective terms were queried and then the trends between their RSVs were compared. Statistical significance was defined as $\mathrm{P}<0.05$ for two-tailed tests. Due to the de-identified nature of the data used in the study, informed consent for this study was not obtained and it was exempt of Institutional Review Board approval. This study was conducted in compliance with all the applicable institutional ethical standards as applicable for human study.

\section{Results}

The RSV from 2004 to 2019 for "Go Red for Women," "heart attack (in) women," "stroke (in) women," and "heart disease (in) women" tended to increase every February (Fig. 1a). Overall, the RSV of "heart attack (in) women" was the highest in all months, outweighed the other three terms by a ratio of $9: 2$, and increased significantly over time. Specifically in February, the RSV of "Go Red for Women" increased on average 494\% (range: $211 \%$ to $789 \%$ ) when compared to the other months of each year with no active campaign. Similarly, February's RSV for "heart disease (in) women" and "heart attack (in) women" increased by $114 \%(24 \%$ to $182 \%)$ and $21 \%(-13 \%$ to $44 \%)$, respectively, but decreased $7 \%$ (-86\% to $20 \%)$ for "stroke (in) women" (Fig. 1b).

The correlation of "Go Red for Women" with "heart disease (in) women" was moderately positive $(\mathrm{r}=0.54, \mathrm{P}<$
$0.001)$, but it was poor with "heart attack (in) women" ( $\mathrm{r}=$ $0.12, \mathrm{P}=0.12)$ and "stroke (in) women" $(\mathrm{r}=-0.03, \mathrm{P}=0.68)$. In contrast, for BCAM, RSV in October for "Breast Cancer Awareness Month" and "breast cancer" increased in average $578 \%$ (range: $238 \%$ to $1,000 \%$ ) and $136 \%(45 \%$ to $206 \%$ ) respectively, and showed high positive correlation $(\mathrm{r}=0.78, \mathrm{P}<$ 0.001 ). Figure 1c illustrates that the RSV of BCAM was higher than GoRedW throughout the 15-year study period (average RSV: 8.89 versus 2.36), and had a higher increase during the active campaign month, $978 \%$ (425\% to $1,805 \%)$ for BCAM and $459 \%$ (200\% to $794 \%$ ) for GoRedW.

\section{Discussion}

This is the first study to evaluate the effect of the GoRedW campaign on online information-seeking patterns. GTr was used because it provides data from a large sample of searches from the most widely used search engine. There was an increase in the RSVs which correlated with the respective active campaign months for GoRedW and BCAM. Our study showed a temporal increase over the 15-year study period where RSV of "Go Red for Women" had a strong increase in February. Among the search queries for CVD the term "heart disease (in) women" had the highest correlation in RSVs associated with the campaign month while the term "heart attack (in) women" also increased substantially. The general increase over time and year-round of searches for "heart attack (in) women" is also noteworthy in our findings. This may suggest that when Internet users search for heart conditions unprompted they use the term "heart attack" as it is a more common term used in non-medical groups, while the GoRedW campaign's use of the term "heart disease" in February may lead to the increased correlation in searches for that term during the campaign month. Accordingly, if knowledge of the disparity in detection and treatment of heart conditions in women is reaching the lay public due to efforts such as the GoRedW campaign, we might expect searches for the term "heart attack (in) women" to increase noticeably over time, as seen in our study.

Interestingly, GoRedW aims to raise awareness of CVD and stroke in women, but the data showed that the campaign month is only associated with higher RSVs for both search terms related to CVD but not to stroke in women. Campaign materials for GoRedW by the American Heart Association (AHA) address both CVD and stroke awareness in women. However, our results could suggest a higher emphasis and more successful public engagement in heart disease advocacy compared to stroke. This can be explained by: 1) The time of GoRedW in the month of February has also been designated by the AHA as American Heart Month, where there is an emphasis on heart health; 2) The prominence of the color red in the campaign name, branding, and events of GoRedW may prompt the public to associate it more with heart conditions than stroke. Further studies could investigate the relative emphasis on CVD and stroke in GoRedW campaign materials and explore additional strategies to increase the effectiveness of the messaging for both aims of the GoRedW initiative.

Furthermore, the RSV of GoRedW was significantly small- 

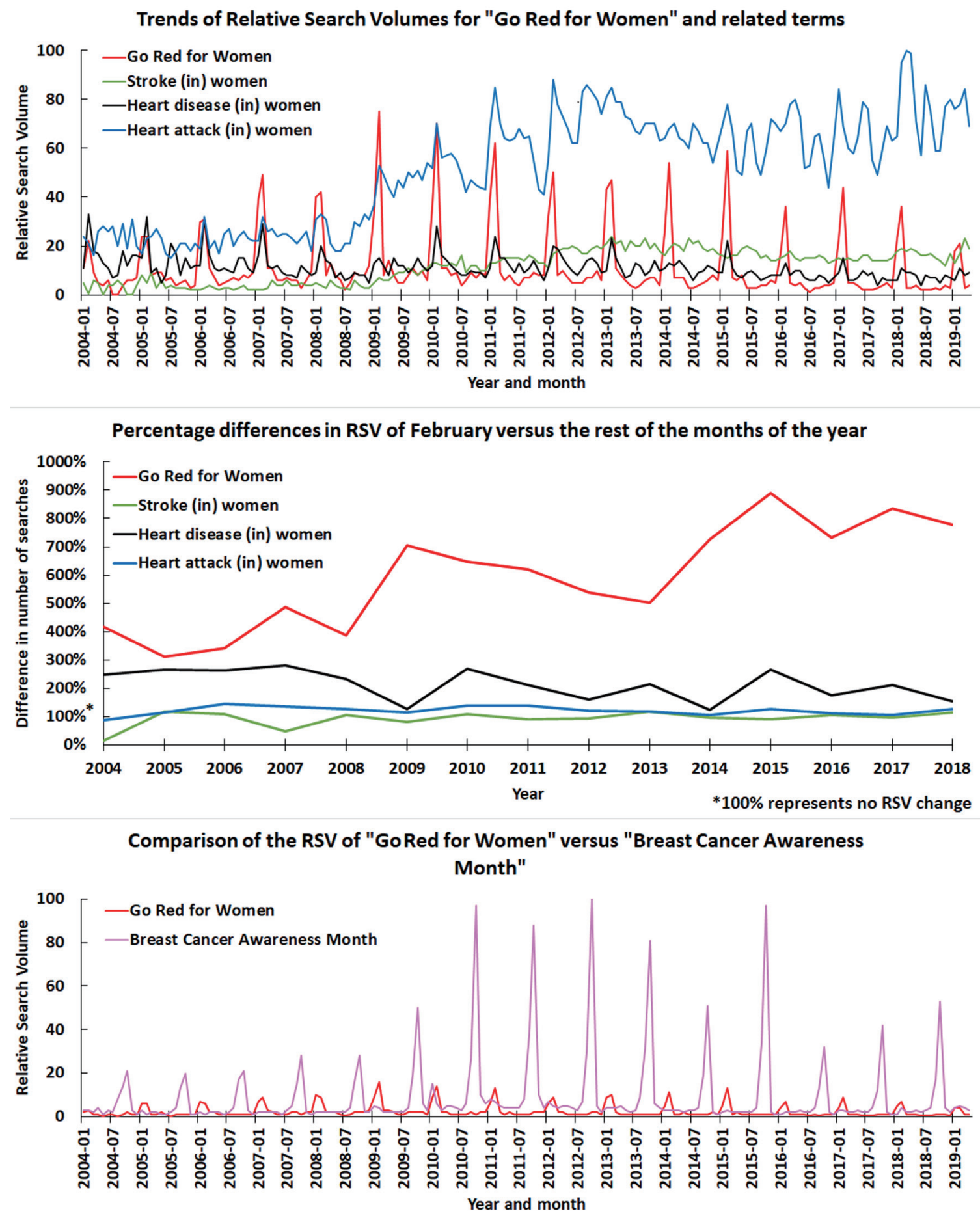

Figure 1. GTr search volume trends for Go Red for Women (a, b) and Breast Cancer Awareness Month campaigns (c). GTr: Google Trends.

er than that of BCAM in their respective campaign months. While this difference serves as a relative indicator of each campaign's effect on the volume of Internet searches, it does not represent a comprehensive evaluation of their overall success (including off-line behaviors) in raising public awareness, which was beyond the scope of this study. Nevertheless, this stark dif- ference in the RSV could reflect differences in the history and social positioning of these campaigns. For instance, BCAM has been addressing breast cancer advocacy since 1985 and has involved several major non-profit organizations in its promotion in the USA. Additionally, many associated initiatives such as "breast cancer walk", "breast cancer NFL" and the "pink ribbon 
culture" have developed around BCAM, permeated popular culture and increased its level of success compared to many other awareness campaigns even for other cancers [11]. Notably, it has also been argued that the high level of interest demonstrated to the BCAM in recent years can be attributed to its pink ribbon culture becoming a target for marketing led by certain promotional industries [11-13].

The limitations of this study must be noted. First, GTr data are not collected with the primary aim of performing health research, but with a commercial aim. GTr can change its datagenerating processes and the results may be similar but not exactly reproducible if the searches are performed on different dates. Second, it should be emphasized that the study subject are the searches themselves, not the people that perform them, and that the search volumes represent normalized data, as GTr does not provide the discrete number of searches. Third, our findings cannot be fully extrapolated to off-line health information seeking and behaviors; they only highlight the effect of awareness campaigns on Internet searches in the most used search engine. Finally, the study only used GTr data, but there are other Internet platforms that may provide insightful information about online information-seeking and informationsharing behaviors such as Twitter Insights and Twitonomy ${ }^{(}$. Although these other platforms have different users and content, they can also be used to inform about the effects of awareness campaigns such as GoRedW.

Of note, the GoRedW and BCAM goals are to increase awareness to reduce mortality related to CVD and breast cancer in women, respectively. However, there is little public awareness of the intersection of these two devastating conditions in women. To this end, a scientific statement by the AHA highlighted connections between CVD and breast cancer due to similar risks factors and the occurrence of cardiotoxicities with breast cancer therapies $[14,15]$. The field of cardio-oncology (or onco-cardiology) has emerged to address the critical need for multidisciplinary cancer care with an approach to preventing and reducing $\mathrm{CV}$ dysfunction in high-risk cancer patients. Given the intersection of CVD and breast cancer, it may be beneficial to support synergistic efforts of the GoRe$\mathrm{dW}$ and BCAM campaigns for even more effective messaging about interventions in cardio-oncology to help provide the best cancer treatments while protecting the heart of these patients.

This study shows that the GoRedW campaign has a positive effect on the search volume of terms related to CVD in woman, but also shows that there are still opportunities for improvement, such as increasing awareness of stroke in women. Efforts to improve awareness of CVD in women are encouraged and needed.

\section{Acknowledgments}

None to declare.

\section{Financial Disclosure}

None to declare.

\section{Conflict of Interest}

The authors have no conflict of interest to disclose.

\section{Informed Consent}

Not applicable.

\section{Author Contributions}

G. A. Suero-Abreu designed the study, engaged in data collection, reviewed the literature, analyzed and interpreted the data, and drafted the manuscript. A. Barajas-Ochoa designed the study, oversaw data collection, reviewed the literature, analyzed and interpreted the data, and reviewed the manuscript. A. Perez-Peralta and E. Rojas contributed to the literature review and provided critical reviews of the manuscript. R. Berkowitz oversaw the design of the study, engaged in data interpretation, and provided critical reviews of the manuscript.

\section{Data Availability}

The authors declare that data supporting the findings of this study are available within the article.

\section{References}

1. Benjamin EJ, Virani SS, Callaway CW, Chamberlain AM, Chang AR, Cheng S, Chiuve SE, et al. Heart disease and stroke statistics-2018 update: a report from the American Heart Association. Circulation. 2018;137(12):e67-e492.

2. American Heart Association. Go red for women [Internet]. 2020 [cited 2020 April 27]. Available from: https:// www.goredforwomen.org/.

3. Reimold SC, Hill JA. Inaugural go red for women issue. Circulation. 2017;135(6):493-494.

4. Mosca L, Hammond G, Mochari-Greenberger H, Towfighi A, Albert MA, American Heart Association Cardiovascular D, Stroke in W, et al. Fifteen-year trends in awareness of heart disease in women: results of a 2012 American Heart Association national survey. Circulation. 2013;127(11):1254-1263, e1251-1229.

5. Senecal C, Widmer RJ, Lerman LO, Lerman A. Association of search engine queries for chest pain with coronary heart disease epidemiology. JAMA Cardiol. 2018;3(12):1218-1221.

6. Martinez-Arroyo G, Ramos-Gomez S, Rojero-Gil EK, Rojas-Gongora JA, Barajas-Ochoa A, BustamanteMontes LP, Yanez J, et al. Potential uses of an infodemiology approach for health-care services for rheumatology. Clin Rheumatol. 2019;38(3):869-876.

7. Eysenbach G. Infodemiology and infoveillance: framework for an emerging set of public health informatics methods to analyze search, communication and pub- 
lication behavior on the Internet. J Med Internet Res. 2009;11(1):e11.

8. Nuti SV, Wayda B, Ranasinghe I, Wang S, Dreyer RP, Chen SI, Murugiah K. The use of google trends in health care research: a systematic review. PLoS One. 2014;9(10):e109583.

9. StatCounter. Search engine market share worldwide [Internet]. 2020 [cited 2020 April 27]. Available from: https://gs.statcounter.com/search-engine-market-share.

10. Google. FAQ about Google Trends data [Internet]. 2020 [cited 2020 April 27]. Available from: https://support. google.com/trends/answer/4365533?hl=en.

11. Glynn RW, Kelly JC, Coffey N, Sweeney KJ, Kerin MJ. The effect of breast cancer awareness month on internet search activity - a comparison with awareness campaigns for lung and prostate cancer. BMC Cancer. 2011;11:442.
12. Sulik GA. Pink ribbon blues. How breast cancer culture undermines women's health. New York: Oxford University Press. 2011.

13. The Lancet Oncology. Breast-cancer awareness: too much of a good thing? Lancet Oncol. 2007;8(12):1041.

14. Mehta LS, Watson KE, Barac A, Beckie TM, Bittner V, Cruz-Flores S, Dent S, et al. Cardiovascular disease and breast cancer: where these entities intersect: a scientific statement from the American Heart Association. Circulation. 2018;137(8):e30-e66.

15. Gilchrist SC, Barac A, Ades PA, Alfano CM, Franklin BA, Jones LW, La Gerche A, et al. Cardio-oncology rehabilitation to manage cardiovascular outcomes in cancer patients and survivors: a scientific statement from the American Heart Association. Circulation. 2019;139(21):e997-e1012. 